\title{
EVALUATION GLOBALE DU TRANSFERT SÉDIMENTAIRE LE LONG DU CORDON LITTORAL DE SÈTE À MARSEILLAN
}

\author{
. M. RADULESCU (1), J.P. BARUSSEAU ${ }^{(2)}$ et F. RUEDA (3)
}

(1) Institut des Aménagements Régionaux et de l'Environnement, Montpellier

(2) Laboratoire de Recherche de Sédimentologie Marine, Université de Perpignan

(3) Service Maritime et de Navigation du Languedoc-Roussillon, Sète

\begin{abstract}
A 6-years sedimentary balance has been allowed by a series of topo-bathymetric cross-sections during 19841990 along the barrier beach of Thau Lagoon between Sete and Marseillan. The recent accumulation tendency recorded in the central part of this costal zone exceeds the estimate resultant southwesterly longshore sediment transport. It could be explained by several hypotheses :

- larger sand supply crossing the rocky bottoms from the $N E$,

- sand supply from the outside of the nearshore zone after destruction of the fossil beachrock barrier at the NE

- important occurrent northeasterly sediment transport from the non-surveyed southwesterly part of the coastal zone

- temporary shoreward sand migration from the offshore in the absence of recent strong storms.
\end{abstract}

\section{Résumé}

Un bilan sédimentaire provisoire de l'avant-côte du cordon littoral de l'étang de Thau entre Sète et Marseillan est rendu possible par le suivi de l'évolution des profils en travers (6 levés topo-bathymétriques périodiques) pendant la période 1984-1990. Le volume réel de l'accumulation enregistrée récemment dans la partie centrale de cette zone côtiere dépasse quantitativement le volume estimé du transit sédimentaire, dont la résultante annuelle est de sens NE-SW. Cet excédent pourrait être expliqué selon plusieurs hypotheses:

- un apport sableux non négligeable traversant les fonds rocheux situés au NE, devant le promontoire de Sete;
- des apports augmentés provenant d'une zone plus profonde après la destruction progressive du banc de grès de plage fossile situé dans la partie $N E$;

- l'occurrence d'un transport littoral important vers le $N E$, provenant du secteur sableux non suivi situé au SW (Marseillan-Plage);

- une migration du sable du large vers la côte. généralement favorisée par l'absence de fortes tempêtes pendant cette période récente.

Une série de 6 profils topo-bathymétriques effectués par le SMNLR de Sète dans 5 sections fixes pendant les années 1984-1990 (octobre 1984, juin 1986, septembre 1988, mai 1989, octobre 1989 et juin 1990) permet un aperçu des changements du microrelief sous-marin intervenus sur l'avant-côte du cordon littoral de l'étang de Thau pendant cette période. Une sixième section a été établie à partir de 1989 entre les profils 15 et 16, afin de combler l'intervalle trop espacé entre ces deux sections de mesures (fig. 1, 15 b).

La planimétrie des surfaces représentant les coupes successives en travers du prisme littoral délimité par un plan vertical correspondant au front du talus de l'arrièreplage et le plan horizontal de lisobathe $10 \mathrm{~m}$ (fig. 2) nous donne la possibilité d'analyser l'évolution des différences de volume unitaire de sédiment (DV, $\mathrm{m}^{3} / \mathrm{m}$ ) dans le temps (dans une section donnée) et dans l'espace (d'une section à l'autre, le long de la côte).

Il est évident que les mouvements des masses sédimentaires dans le profil transversal sont concentrées dans la zone des barres sableuses d'avant-côte et d'avantplage, les variations enregistrées au large de cette zone étant de moindre ampleur. Ainsi, les deux barres principales, telles qu'elles étaient situées en 1984, sont

\begin{tabular}{|c|c|c|c|c|c|c|c|c|c|c|c|}
\hline $\begin{array}{l}\text { Date } \\
\text { Profil }\end{array}$ & 30.10 .84 & & 06.86 & & 27.09 .88 & & 30.10 .89 & & 12.06 .90 & $\Delta V(84-90)$ & $\Delta V / a n$ \\
\hline & $\Delta V_{L}$ & $\Delta V_{T}$ & $\Delta V_{L}$ & $\Delta \mathrm{V}_{\mathrm{T}}$ & $\Delta V_{L}$ & $\Delta \mathrm{V}_{T}$ & $\Delta V_{L}$ & $\Delta V_{T}$ & $\Delta \mathrm{V}_{\mathrm{L}}$ & & \\
\hline 17 & & $+230,0$ & & $-270,0$ & & $+15,0$ & & $-82,5$ & & $-107,5$ & $-17,9$ \\
\hline & $+135,0$ & & $+225,0$ & & $+160,0$ & & $+175,0$ & & $+235,0$ & & \\
\hline 16 & & $+100,0$ & & $-115,0$ & & +30 & & $-22,5$ & & $-7,5$ & $-1,25$ \\
\hline & $+232,5$ & & $+295,0$ & & $+417,5$ & & $+285,0$ & & $+357,5$ & & \\
\hline 15 & & $+412,5$ & & $-242,5$ & & $-102,5$ & & +50.0 & & $+117,5$ & +19.6 \\
\hline & (?) & & $+127,5$ & & $+257,5$ & & $+262,5$ & & $+270,0$ & & \\
\hline 14 & & (?) & & $-112,5$ & & $-97,5$ & & $+57,5$ & & (?) & (?) \\
\hline & (?) & & $\begin{array}{r}-55,0 \\
\end{array}$ & & $-245,0$ & & $-57,5$ & & $-35,0$ & & \\
\hline 13 & & $+357,5$ & & $-302,5$ & & $+90,0$ & & $+80,0$ & & $+225,0$ & $+37,5$ \\
\hline
\end{tabular}

$\Delta V_{L}=$ le long du listoral, entre les sections ; $\Delta V_{T}=$ dans le temps, dans la même section.

Tabl. 1 : Différences de volume unitaire $\left(\mathrm{m}^{3} / \mathrm{m}\right)$ entre les profils topo-bathymétriques en travers de la zone littorale de Sète à Marseillan pendant la période 1984-1990 
remplacées en 1990 par les fosses inter-barres, avec un maximum de variation de profondeur de $4 \mathrm{~m}$ dans le plan vertical (fig. 2).

En examinant les valeurs présentées dans le tableau 1, on remarque un hiatus produit par l'absence de données bathymétriques dans le profil 14 en 1984, sans influence sur les résultats de l'analyse, comme on verra plus loin. Dans les colonnes, les sections standard sont insérées dans l'oräre du nord-est (Sète) vers le sud-ouest (Marseillan), suivant la direction prédominante du transit littoral de sédiments le long de cette côte. Rangées sur l'horizontale, les dates des levés montrent une inconséquence plus importante : tous les deux ans, au début du suivi, les profils ont été effectués annuellement pendant les derniers trois ans (voire deux fois par an en 1989) ; des profils d'été alternent avec des profils d'automne, ce qui pourrait aussi influencer légèrement les résultats. Pour l'année 1989, les données acquises au mois d'octobre ont été préférées dans le calcul des valeurs DV à celles de mai, susceptibles de correspondre à un régime plus dynamique et instable par rapport aux circonstances de l'ensemble des sondages.

L'analyse de la variation des paramètres DV dans le temps et le long du cordon littoral met en évidence quelques particularités. Pour les valeurs $\mathrm{DV}_{\mathrm{T}}$ :

- une croissance générale entre 1984 et 1986, due apparemment aux remontées de sédiments enlevés par les tempêtes de 4 octobre 1984 (juste avant le levé des profils) et des années précédentes sur la pente du plateau continental (fig. 2) ;

- une décroissance générale entre 1986 et 1988 , intervalle dans lequel la forte tempête de 4-5 décembre 1987 a eu un impact important sur les fonds marins de l'avant-côte languedocienne ;

- des valeurs négatives dans la partie NE (profils 17 et 16) associées à des valeurs positives dans les profils situés vers le SW, dans le sens du transfert littoral résultant, entre 1989 et 1990, un intervalle sans événements hydrométéorologiques majeurs.
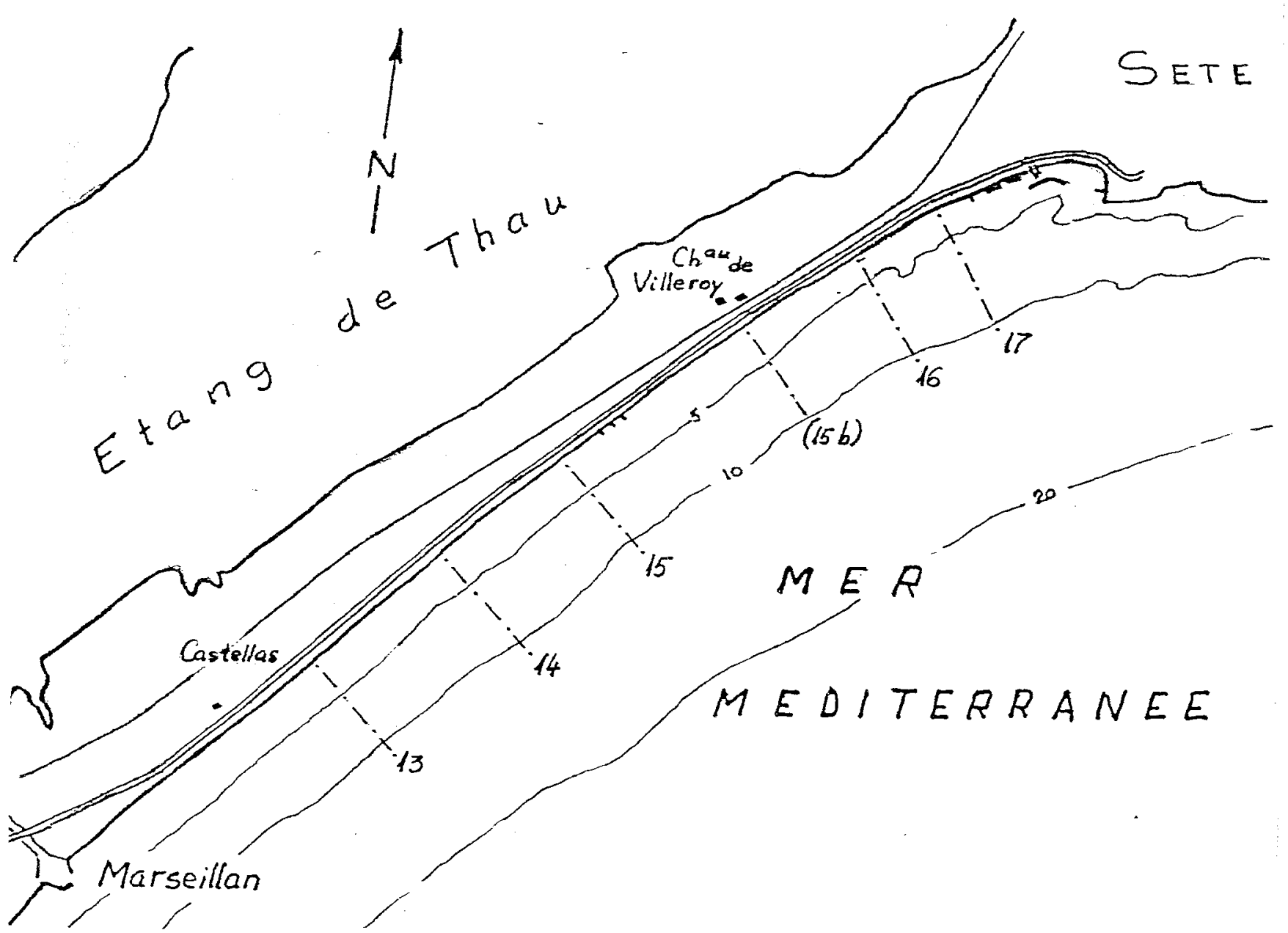

Figure 1 - Plan de situation des profils bathymétriques

La distribution des paramètres $D V_{L}$ dans le sens NE SW montre des valeurs toujours positives entre les profils 17 à 14 et toujours négatives entre les profils 14 et 13, traduisant l'existence dans toutes les circonstances hydrodynamiques d'une dorsale plus élevée sur les fonds sableux de l'avant-côte au niveau du profil 14 , comme une protubérance accumulative alimentée par des apports latéraux convergeant dans les deux sens. L'équilibre dynamique de la plage émergée dans ce secteur, mis en évidence par une série de 12 levés topométriques entre 1987 et 1993, confirme cette particularité morphologique locale de la zone côtière. 

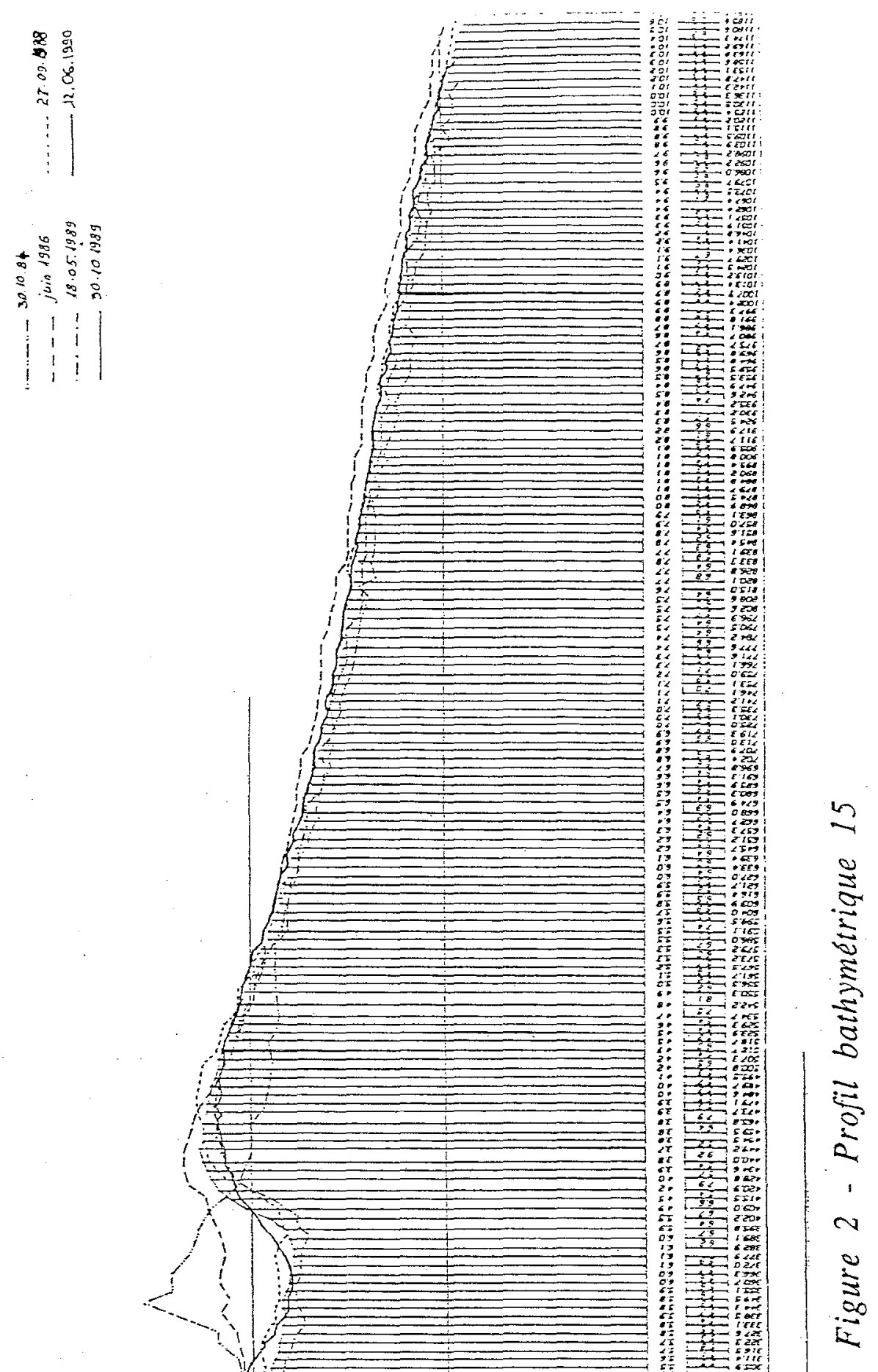
La période courte d'observation (6 ans) et l'inconstance révélée plus haut dans la périodicité de l'échantillonnage recommandent les valeurs du taux d'évolution du stock sédimentaire calculées dans la dernière colonne comme provisoires à l'échelle du temps. Au contraire, la distribution spatiale de ces paramètres le long du cordon littoral leur conferent une certaine signification relative. La croissance uniforme des valeurs $\mathrm{DV}_{\mathrm{T}}$ cumulées sur la période 1984-1990 entre les profils 16 et 13 est représentée graphiquement dans la figure 3 . Ces valeurs cumulées sont négatives seulement dans les profils 16 et 17, qui traversent une zone en érosion, partiellement protégée par des ouvrages en mer. Quant au bilan de la zone à tendance accumulative qui commence au sud d'un point neutre situé au sud du P16, il est équivalent, pour cette période, à un dépôt de $720000 \mathrm{~m}^{3}$ et les apports de sédiments ne peuvent pas être fournis uniquement par l'érosion du secteur NE, beaucoup plus réduit (la côte devient entièrement rocheuse à proximité de Sète).

D'une façon générale, l'augmentation NE-SW du taux d'accumulation sur les hauts fonds de l'avant-côte correspond à un gradient négatif du transit sédimentaire longitudinal dans cette direction (SOGREAH, 1985). Cependant, le bilan dépasse largement le débit potentiel de sédiments, estimé pour les sections NE à 30000 $\mathrm{m}^{3} / \mathrm{an}$.

En conclusion, si on fait abstraction du nombre pour le moment réduit de données bathymétriques, plusieurs hypothèses pourraient expliquer ce bilan positif dans la partie centrale du cordon littoral :

- un transit sédimentaire potentiel plus fort sur les fonds rocheux situés à l'extrémité NE, permettant des apports supplémentaires de la zone de contact, plus profonde, entre les rochers et les sables,

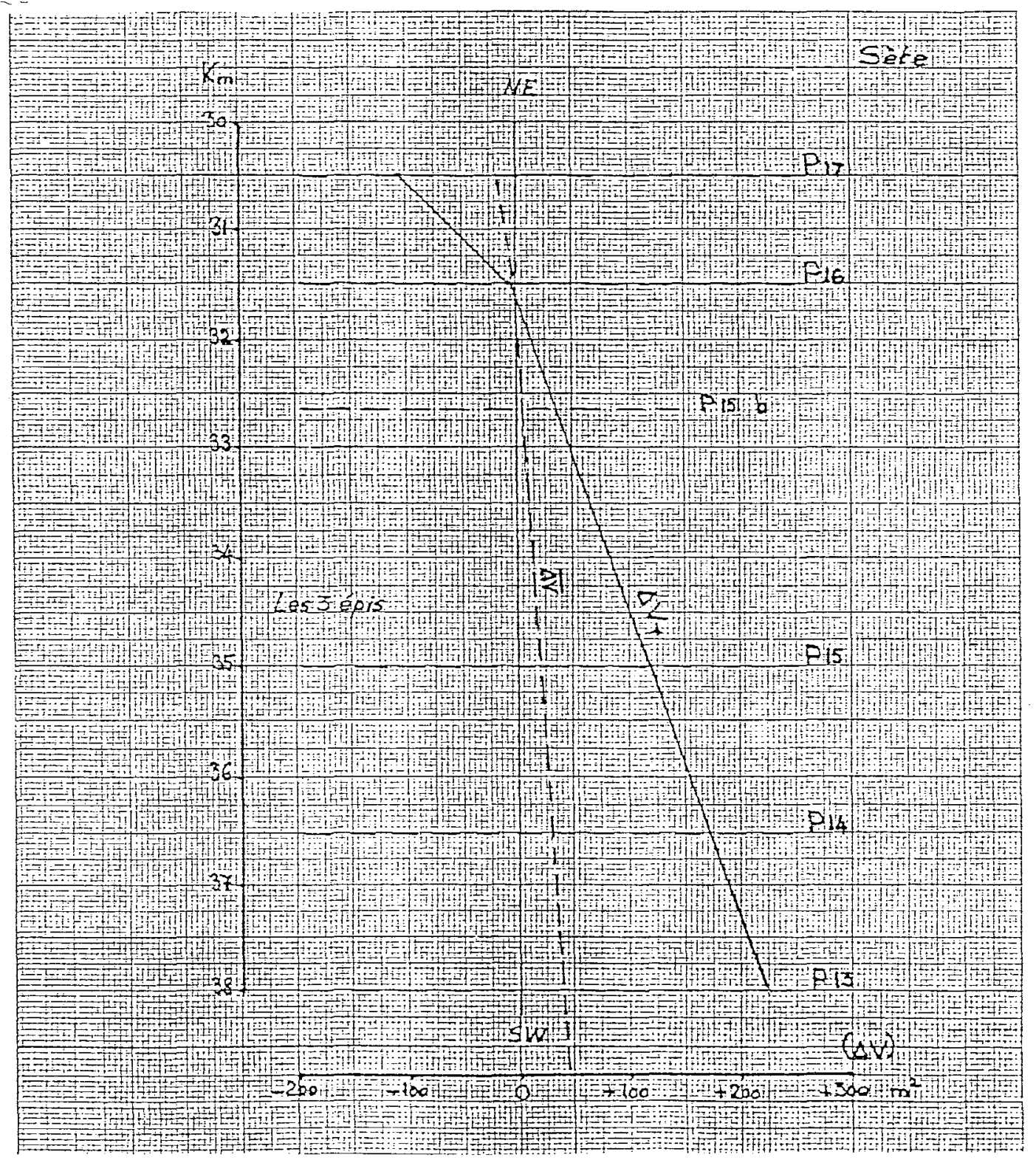

Figure 3 - Evolution du paramètre DV le long du cordon littoral 
- un apport de sable de l'extérieur de la zone de déferlement des vagues, déstabilisé dans la partie NE par un déblaiement progressif (constaté) du cordon fossile de beachrock ;

- des apports importants de $S W$, avec un gradient négatif du transit vers la partie centrale du cordon littoral (P14),

- une temporaire remontée générale de sable fin mis en mouvement par fortes houles à des profondeurs de plus de $10 \mathrm{~m}$ en l'absence, dernièrement, de très fortes tempêtes.

Il faut rappeler que les différences de stocks sableux cantonnés instantanément dans différentes sections ne représentent qu'une résultante d'une multitude de mouvements sédimentaires dont le sens n'est pas toujours NE-SW. Même si à une certaine échelle du temps, pour des périodes sans tempêtes exceptionnelles, on peut négliger les échanges, en général plus faibles, avec la zone située au-delà de l'isobathe $10 \mathrm{~m}$, le prolongement du suivi vers le $S W$, jusqu'aux digues de l'avant-port de Marseillan, apparait absolument nécessaire pour une évaluation du transfert sédimentaire résultant le long de ce cordon littoral par une méthode globale.

\section{Références}

Ruéda F. 1985. Le littoral de l'Hérault et du Gard

Radulescu M., Barusseau J.P., Gerbe A. 1990. Contribution à l'étude de la dynamique sédimentaire de la plage de Sète Comptes rendus du $1^{\text {er }}$ symposium international de l'Assoriation Eurocoast , 9-13 juillet 1990, Marseille.

SOGREAH. 1985. Protection de la plage de Sète. Etude diagnostic, $450085 \mathrm{R} 2$.

\section{QUESTION DE MONSIEUR CARBONNEL :}

En liaison avec l'exposé précédent présenté par Monsieur Barusseau (évolutions du profil transversal), vous a-t-il été possible d'exploiter les nombreux profils bathymétriques mensuels, et si ce n'est pas le cas cela n'aurait il été pas souhaitable?

Le problème étant la comparaison de profils de plage séparés de 2 ans environ, à des saisons différentes, et dont les conditions météorologiques préalables ne sont pas à priori connues.

Les conclusions de variation de volume de sable constatés seraient donc à manier avec beaucoup de prudence, en les corrélant sans doute avec d'autres données ou réflexions sur le site.

\section{REPONSE}

Les types de résultats que l'on peut attendre d'une observation à pas de temps long ou à pas de temps court des profils de plage ne sont pas les mêmes en raison de rythmes interannuels superposés aux rythme saisonniers et mensuels. Les deux approches sont utiles et sont présentées respectivement dans les deux communications. 